\title{
Article \\ A Human-Inspired Control Strategy for Improving Seamless Robot-To-Human Handovers
}

\author{
Paramin Neranon * a and Tanapong Sutiphotinun
}

check for updates

Citation: Neranon, P.; Sutiphotinun, T. A Human-Inspired Control Strategy for Improving Seamless Robot-To-Human Handovers. Appl. Sci. 2021, 11, 4437. https://doi.org/ 10.3390/app11104437

Academic Editor: Antonio

Fernández-Caballero

Received: 20 April 2021

Accepted: 10 May 2021

Published: 13 May 2021

Publisher's Note: MDPI stays neutral with regard to jurisdictional claims in published maps and institutional affiliations.

Copyright: (c) 2021 by the authors. Licensee MDPI, Basel, Switzerland. This article is an open access article distributed under the terms and conditions of the Creative Commons Attribution (CC BY) license (https:/ / creativecommons.org/licenses/by/ $4.0 /)$.
Department of Mechanical and Mechatronics Engineering, Faculty of Engineering, Prince of Songkla University, Songkhla 90112, Thailand; Tanapong_S2539@hotmail.com

* Correspondence: paramin.n@psu.ac.th

\begin{abstract}
One of the challenging aspects of robotics research is to successfully establish a humanlike behavioural control strategy for human-robot handover, since a robotic controller is further complicated by the dynamic nature of the human response. This paper consequently highlights the development of an appropriate set of behaviour-based control for robot-to-human object handover by first understanding an equivalent human-human handover. The optimized hybrid position and impedance control was implemented to ensure good stability, adaptability and comfort of the robot in the object handover tasks. Moreover, a questionnaire technique was employed to gather information from the participants concerning their evaluations of the developed control system. The results demonstrate that the quantitative measurement of performance of the human-inspired control strategy can be considered acceptable for seamless human-robot handovers. This also provided significant satisfaction with the overall control performance in the robotic control system, in which the robot can dexterously pass the object to the receiver in a timely and natural manner without the risk of harm or injury by the robot. Furthermore, the survey responses were in agreement with the parallel test outcomes, demonstrating significant satisfaction with the overall performance of the robot-human interaction, as measured by an average rating of 4.20 on a five-point scale.
\end{abstract}

Keywords: object handover; robot-to-human object handover; human-robot interaction; humanhuman interaction; hybrid position/force control; impedance control; human-like behavioural control

\section{Introduction}

Recently, robots have been widely developed over the last decades to meet the requirements of improving human-robot interaction (HRI). HRI is a field of study to understand, design and evaluate robotic systems, while interacting with humans [1]. The interaction involves several communication forms and can be mainly categorized into two types: remote interaction (i.e., search and rescue [2-4], military and police [5,6], space exploration [7-9], etc.) and proximate interaction (i.e., assistive robotics and educational robotics [10-14], home or industry $[15,16]$, etc.).

In this research, the field of social robotics and human-robot interaction are highlighted, in which robots are being developed to serve in human assistive capacities. One of the main challenges in close proximity interaction is to efficiently establish a framework for seamless human-robot handovers (HRH). This will allow a human to act jointly with a robot safely and reliably. The researchers [17] claimed that understanding of behavioural control strategies of human-human interaction (HHI) is fundamental to design an effective HRI system. In addition, Neranon et al. [18] also addressed that understanding the principle of human haptic interaction, when two humans work together in a joint effort to complete a shared task, is crucial in designing an effective HRI system.

This section emphasizes the background of handover-based research, since humanhuman handovers $(\mathrm{HHH})$ are the ultimate benchmark for seamless handovers. A giver is defined as an agent who holds an object and passes it to another. In the physical interaction, a receiver is a particular position that receives the object by starting pulling it. Afterwards, 
the receiver takes responsibility for the object throughout the transferring process $[19,20]$. Much of the relevant HHI research, which has steadily increased to enhance the understanding of HHI behaviour, has been reviewed. Previous studies have examined issues regarding investigating the control characteristics of two humans in a physical handover task, for examples, analysing (1) the influence of velocity [21,22] and trajectory [23,24] on the handover movements, (2) grip forces and load forces in the object exchange [25], (3) grasp points [26,27] and locations [28,29] and finally, (4) configuration [30,31] of the exchanged object. These can be used as conceptual frameworks for designing control systems for cooperative robots to work with other partners by imitating human-based behaviour control strategies.

Sutiphotinun et al. [32] investigated the human behavioural responses in $\mathrm{HHH}$ tasks under various conditions. The paradigm findings show human dynamic characteristics, i.e., interactive force analysis, suitable transfer locations between the givers and receivers and the handover sequences. The handover is distinctively categorized into sendingtransferring-receiving phases, where the giver agent primarily decides to release the object. This will be useful for developing a robotic behaviour-based approach in seamless HRH in future. Saki Kato et.al [33] analysed how humans (givers and receivers) determine the locations of object transfer during handovers for a good understanding of the mechanisms of human social behaviour. The study also presented a linear model with the influential factors affecting the handover locations. It consists of participant heights, genders and social dominance of the two partners and the distance between both humans.

Chien et al. [34] proposed adaptive coordination strategies for physical HRH. It was based on the identification of $\mathrm{HHH}$ strategies regarding how people adapt their handover actions to the workload of their partners. The newly developed approach involving two baselines, namely, (1) proactive and (2) reactive coordination techniques, enabled similarly fluid and effective HRH tasks. Sisbot et al. [35] proposed a robotic navigation planner and a manipulation planning framework by first understanding the human dynamic characteristics of the haptic interaction. This facilitated the robot to autonomously select the best handover location based on context (i.e., accessibility, posture, a field of view and human's safety etc.). This offered safe, legible and socially acceptable paths for HRH tasks. Some researchers [36-38] examined a robotic mechanism guideline to allow the robot to grasp an object based on human-like reaching gestures before handing it over to a human partner. It involved the object's shape and function and the minimization of the risk of an injury occurring during the HRI execution.

Jae-Bong et al. [39] developed a general-purpose software framework for a Human support robot (HSR) service robot. It directly communicated with a human using complex speech commands. This allowed the robot to move smoothly in a confined space and also manipulate various household objects and autonomously pass them to a human. As extensively reviewed, the existing research on the development of $\mathrm{HRH}$ has almost underlined the investigation on behavioural strategies and the establishment of a framework for a robotic behaviour-based approach. This allowed the robot to approach a person, reach over an object and hand over it with human-like configurations and motions.

However, there has been little discussion on the crucial challenges of the development of seamless HRI, since a robotic control system is further complicated by the dynamic nature of the human environment. Thus, it necessitates a very careful design and implementation of optimized behavioural control strategies to significantly improve the success rate of effective object handover tasks. This also protects a human partner from the risk of harm or injury by a robot in the cooperative tasks. To solve the limitations as mentioned, therefore, this paper presents the design of a human-inspired control strategy for improving seamless robot-human handovers using the hybrid PID position control and impedance control algorithm. This system will enhance the accuracy of positioning and force regulating abilities in the HRH system and also increase the smoothness of human-robot physical interaction. 
This paper details an optimized robotic control implementation for HRH by first understanding haptic dynamic interaction in $\mathrm{HHH}$ tasks in terms of interactive force, suitable transfer locations between the givers and receivers and the handover sequences. This knowledge is then used to design a robotic behaviour-based approach, which is the crucial aspect of the development of effective HRH. It is followed by how to develop optimized hybrid position/force control involving PID position control and force impedance control. The robotic control incorporates feedback signals from the HSR-3D-depth sensor, encoders (joint estimators) and multi-axis force/torque sensor attached to the robot wrist joint, respectively. The dynamic mechanical model of the physical HRH was additionally explained. Afterwards, the final sections express the control system evaluations, experimental results of the robotic HRH tasks and finally, conclusion.

\section{Design of the Robotic Human-Inspired Control Strategy for HRH}

\subsection{Background of Human-Human Handover Strategy}

It is crucial to understand the kinematics and dynamics of HHH behaviour to design a conceptual guideline for a robotic human-like controller for robust, behaviour-based, $\mathrm{HRH}$. Consequently, a set of HHH tests was initially conducted as fully detailed in our previous journal article [32]. It can be summarized that the experimental results of $\mathrm{HHH}$ intensively addressed (1) investigation of the human handover strategies of the givers and receivers (2) examination of the interactive forces and the transfer locations between the couple and (3) clarification of the mathematical model of the giver's arm, which leads to a better understanding how the giver regulates the bilateral force before releasing the object to be transferred to the receiver.

The tests give the behavioural explanation of $\mathrm{HHH}$ in which the HHH process can be interpreted as three distinct phases consisting of (1) sending, (2) transferring and (3) receiving. In the sending session, the giver starts grasping and holding an object and moves to the receiver, who is in the field of the giver's view. Once the handler recognizes a receiver and his/her position, the giver subsequently computes and estimates a suitable object transfer point based on human-relevant parameters. In the meantime, the giver is then moving to the transfer point facing the receiver before naturally handling the object. The handover locations $y$ (height from the ground) is formulated as Equation (1), where $x_{1}$ and $x_{2}$ are the giver's and receiver's heights, respectively. Contrastingly, the transfer distance (in the $\mathrm{x}$-axis) along the facing direction was approximately $0.85 \mathrm{~m}$.

$$
y=8.23+0.243 x_{1}-9.592 x_{2}-1.626 x_{1}^{2}+1.441 x_{2}^{2}+3.189 x_{1} x_{2}
$$

In the transferring phase, the giver's decision is based on the amount of threshold (interactive) force $\left(f_{\text {tsh }}\right)$, which was significantly proportional to the object mass. The relationship between the $f_{t s h}$ as a function of object mass $\left(\vartheta_{m}\right)$ can be exposed in Equation (2).

$$
f_{\text {tsh }}=-85 \vartheta_{m}{ }^{3}+100.75 \vartheta_{m}{ }^{2}-35.78 \vartheta_{m}+6.75
$$

The handover time was approximately $0.46 \mathrm{~s}$. Moreover, to gain more understanding of how the giver regulates the interactive force, the transfer function $\left(T_{G}\right)$ of the giver's hand can be expressed as the following equation:

$$
T_{G}=\frac{-2.63 \times 10^{-4} S+5211}{S^{2}-20.25 S+51.76}
$$

These findings disclose the human characteristics of the haptic handover interaction and for the aim of obtaining a conceptual guideline for a robotic human-like control strategy in $\mathrm{HRH}$. 


\subsection{Toyota HSR Platform}

An HSR home service as depicts in Figure 1a has been launched by the Toyota Corp. the robot can be controlled intuitively using voice commands or a touchscreen graphical user interface. The core technologies of the HSR comprise speech recognition, object recognition, autonomous navigation and localization, motion planning, task planning, etc. These are adequate to develop HRH in this research. It is realizing that the safety issue is an essential aspect of a robot to collaborate with humans in a safe and natural manner. Obstacle avoidance and a force relaxation mechanism were employed to protect humans from any mistakes or accidents. As the robot has to accommodate a wide variety of household activities, then its cylindrical telescoping body was originally fabricated. It was equipped with a differential pseudo-omnidirectional wheel and a central yaw joint which allow the robot to handle most manipulation tasks with smooth changes in direction [39].

To construct a robot map and map-based self-localization, the robot base has equipped with two types of sensors, i.e., a LIDAR sensor and an inertial measurement unit (IMU). The HSR manipulator arms are made up of a shoulder pitch joint and roll-pitch-roll wrist joints. The robot end-effector has a two-finger parallel gripper (driven by a single motor), which was furnished by a wrist multi-axis force/torque sensor and hand camera. The fingertips were designed to be compliant with the shape of the objects, as it is covered by elastic materials. The robot arm is attached to the body with a three-stage prismatic waist joint, which enables the height of the robot's shoulder to a telescope. The robot's head has been attached by an ASUS Xtion RGBD camera, two RGB cameras, a microphone array and a small display monitor [39-41].

The robot has two onboard computers. The main computer is preserved for Toyota's proprietary code to execute sensor and motor interfaces, motion planning and control and the web-based remote user interface etc. Nevertheless, both computers do not have much computing power for the development of advanced software algorithms. Then, it requires an external computer that can directly communicate with the HSR software platform using Robot Operating System (ROS). The robot software architecture can be categorized into two layers: (1) real-time control and (2) intelligence layers. The first layer executes real-time processing for motor control using motor drivers and servo amplifiers. The second layer deals with analysing or systematically extracting big data such as RGB images and point clouds, in which Linux (Ubuntu) is adopted to run the OS and ROS [39-41]. The HRS software architecture is illustrated in Figure 1b.

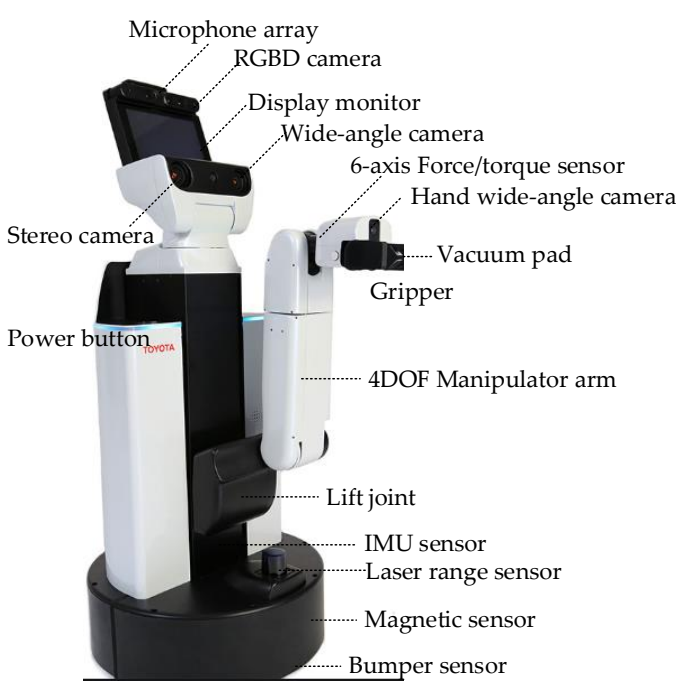

(a)

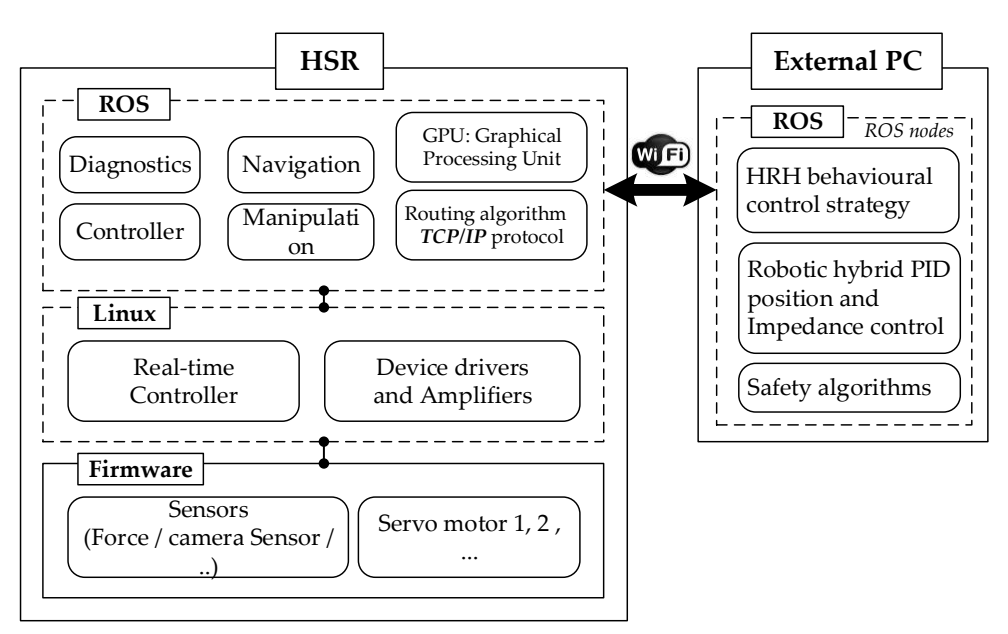

(b)

Figure 1. (a) The hardware equipment of the HRS and (b) HRS software architecture (connecting to an external PC). 


\subsection{The Conceptual Framework for Human-Robot Handover}

The conceptual outline for HRH presents in Figure 2. Based on the human-like behavioural guideline, it can be summarized that the robot initially takes an order given by a receiver; for example, grasping a bottle of water, etc. Then, the robot moves to the object location and extracts the desired object (i.e., using YOLO). The computational system creates a grasping point of the object based on the centre of gravity. Afterwards, the robot plans the hybrid base/arm trajectory using the hybrid inverse kinematics explained in Section 2.4. Once the robot gripper reaches the object, it starts grabbing it with a proper grasping force measured by force sensors attached to the robot fingertips. The robot moves back to the receiver and simultaneously recognize the receiver and his/her location using the head_pose_estimation package detailed in Section 2.5. Subsequently, the robot moves to a transfer location computed using Equation (1). The transferring phase is activated once the receiver manipulates the hand and grasps the object. In the meantime, the service robot has to regulate the bilateral force before releasing the object to be transferred to the receiver. This computational process incorporates the required maximum interactive force formulated in Equation (2). Then, the final phase involves the receiver taking responsibility for the object and the robot retrieving the arm and returning to the home position.

This paper focuses on the design and development of the robot human-like dynamic control, particularly in the transferring session. This will enable similarly fluid and effective object handover tasks with the receiver. Therefore, for all HRH tests, it can be assumed that the robot correctly holds the required object and stays at a home position already. It is ready to promptly handling the object to a human at a defined transfer location.

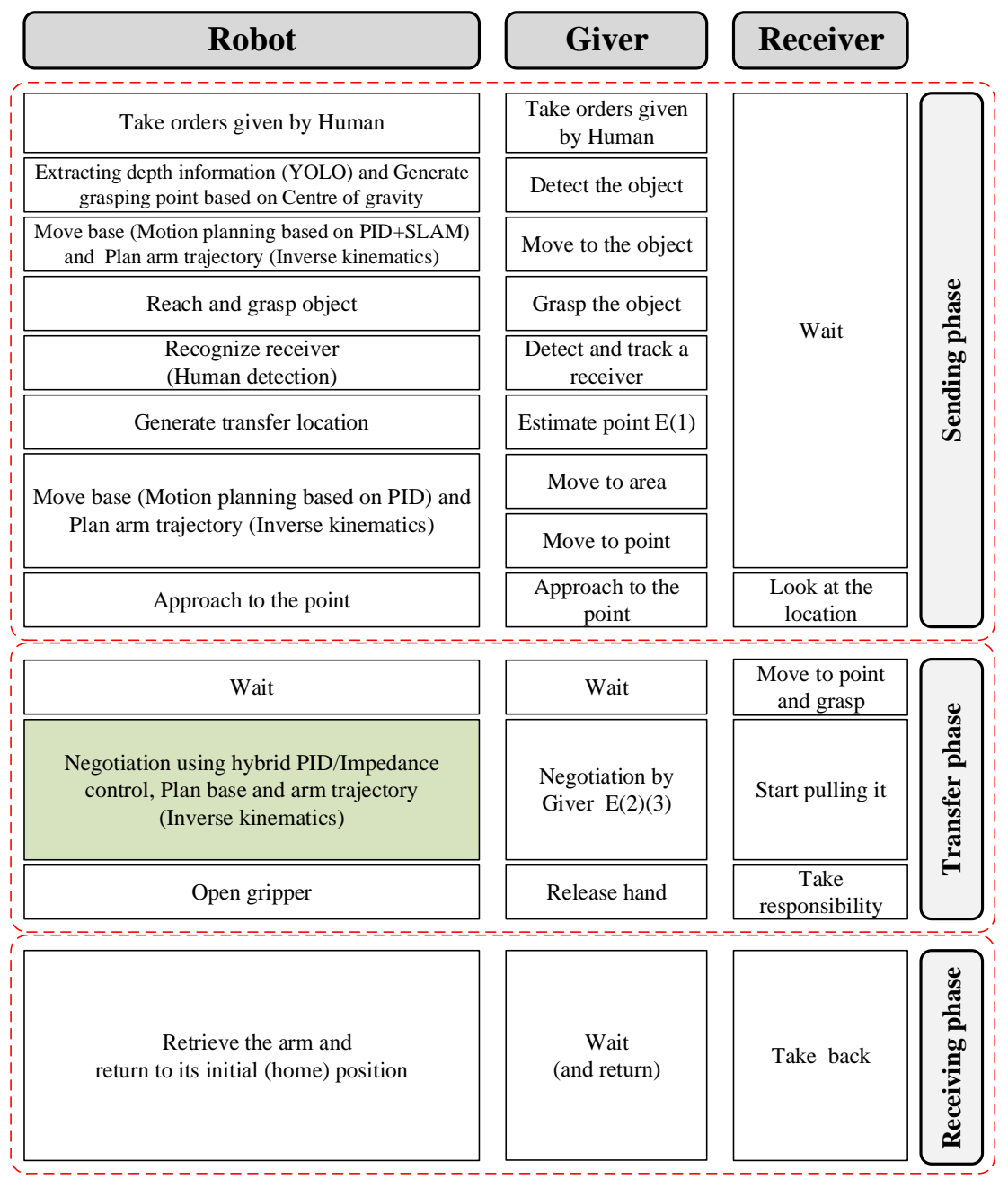

Figure 2. Robot handover framework based on the human-human handover. 


\subsection{Kinematic Model of the HSR}

This section briefly explains the kinematic model of the HSR, which relates to forward and inverse kinematics. In the case of the forward kinematics, the location and pose of the robot end-effector are controlled by the given links and joints of the HSR robot. The Denavit-Hartenberg parameters evaluation is used to achieve the kinematic chain of the robot rigid bodies. Based on the studies by researchers [39-41], the mechanical schematic diagram of a dual-wheel caster-drive robot base and the robot's joint configuration is shown in Figure 3a. The following notation is used in this analysis: the radius of each wheel $(r)$, the angle of the pivot axis $\left(\theta_{H}\right)$, the wheel tread $(W)$, the displacement between the centre axis and the axle centre $(H)$, the inverse kinematics $(f)$, the reference value of the tip pose $\left(T_{e}\right)$, the degree of freedom of the base $\left(\theta_{2}\right)$, the torso lift height $\left(l_{3}\right)$, the shoulder pitch angle $\left(\theta_{4}\right)$, the length of arm $\left(l_{52}\right)$, the length of the wrist $\left(l_{82}\right)$ and finally, the joint angles of the arm $\left(\theta_{5}, \theta_{6}, \theta_{7}\right)$. These are defined in Figure $3 b$.

The robotic dynamic model is essential for describing the HSR robot's position, orientation and motions of the wheels. It can be used for analysing and synthesizing the overall dynamic behaviour of the robot. The robot itself has three degrees of freedom on the $x-y$ plane and can perform forward-reverse movement and left-right movements. As reviewed [39-41], the kinematic equations and relations between robot velocity and various geometry specifications are expressed by the Transformation matrices from linear velocity on the global frame to linear on the robot frame as the following.

$$
\left[\begin{array}{c}
\omega_{R} \\
\omega_{L} \\
\omega_{H}
\end{array}\right]=\left[\begin{array}{ccc}
\frac{r}{2} \cos \theta_{H}-\frac{r H}{W} \sin \theta_{H} & \frac{r}{2} \cos \theta_{H}+\frac{r H}{W} \sin \theta_{H} & 0 \\
\frac{r}{2} \sin \theta_{H}-\frac{r H}{W} \cos \theta_{H} & \frac{r}{2} \sin \theta_{H}-\frac{r H}{W} \cos \theta_{H} & 0 \\
\frac{r}{W} & \frac{r}{W} & 1
\end{array}\right]^{-1}\left[\begin{array}{l}
\dot{x} \\
\dot{y} \\
\dot{\theta}
\end{array}\right]
$$

By assuming that translational and rotational transforms on $x-y-z$ axes are represented as $T_{t}(x, y, z), R_{x}, R_{y}, R_{z}$ and $R_{z}$, respectively, forward kinematics of the HSR arm ( $\left.T_{H S R}\right)$ can be written as:

$$
T_{H S R}=R_{z}\left(\theta_{2}\right) T_{e}\left(0,0, l_{3}\right) R_{y}\left(\theta_{4}\right) T_{e}\left(0,0, l_{52}\right) R_{z}\left(\theta_{5}\right) R_{y}\left(\theta_{6}\right) R_{z}\left(\theta_{7}\right) T_{e}\left(0,0, l_{82}\right) .
$$

According to the kinematic of the HSR arm, the base yaw angle $\left(\theta_{2}\right)$ can be calculated using the relative wrist position $(x, y$ and $z)$ as:

$$
\begin{gathered}
{\left[\begin{array}{llll}
x & y & z & 1
\end{array}\right]^{T}=f T_{e}\left(0,0,-l_{82}\right)\left[\begin{array}{llll}
0 & 0 & 0 & 1
\end{array}\right]^{T}} \\
\theta_{2}=\operatorname{atan} 2(y, x)
\end{gathered}
$$

Analysing torso lift height $\left(l_{3}\right)$ and shoulder pitch angle $\left(\theta_{4}\right)$ gives:

$$
\left[\begin{array}{l}
l_{3} \\
\theta_{4}
\end{array}\right]=\left[\begin{array}{c}
z-\sqrt{l_{52}^{2}-x^{2}-y^{2}} \\
\arcsin \left(\sqrt{x^{2}+y^{2}} / l_{52}\right)
\end{array}\right] \text { or }\left[\begin{array}{l}
l_{3} \\
\theta_{4}
\end{array}\right]=\left[\begin{array}{c}
z-\sqrt{l_{52}^{2}-x^{2}-y^{2}} \\
\pi-\arcsin \left(\sqrt{x^{2}+y^{2}} / l_{52}\right)
\end{array}\right] \text {. }
$$

Investigating the joint angles of the arm $\left(\theta_{5}, \theta_{6}, \theta_{7}\right)$ based on the matrix $T_{R}$ offers:

$$
\begin{gathered}
T_{R}=T_{e}\left(0,0,-l_{52}\right) R_{y}\left(-\theta_{4}\right) T_{e}\left(0,0,-l_{3}\right) R_{z}\left(-\theta_{2}\right) f T_{e}\left(0,0,-l_{82}\right), \\
{\left[\begin{array}{c}
\theta_{5} \\
\theta_{6} \\
\theta_{7}
\end{array}\right]=\left[\begin{array}{c}
\operatorname{atan} 2\left(R_{23}, R_{13}\right) \\
\arccos \left(R_{33}\right) \\
\operatorname{atan} 2\left(R_{32},-R_{31}\right)
\end{array}\right]}
\end{gathered}
$$

However, to allow the HSR to interact naturally with a human and to facilitate the dexterous transfer of objects promptly, hybrid inverse kinematics proposed by Yamamoto et al. [41] was implemented. It involves the inverse kinematics for the eight-DOF HSR including the robot base with three DOF and the arm with five DOF. The hybrid inverse 
kinematics $(f)$ for the HSR can be formulated using the robot tip pose $\left(T_{e}\right)$ as in the following equation:

$$
\left(\theta_{0}, \theta_{1}, \theta_{3}, \theta_{5}, \theta_{6}, \theta_{7}\right)=f\left(T_{e}, \theta_{2}, \theta_{4}\right)
$$

Given $\theta=\left(\theta_{0}, \theta_{1}, \theta_{2}, \theta_{3}, \theta_{5}, \theta_{6}, \theta_{7}\right)$ and the desired reference joint angles $\left(\theta^{r e f}\right)$ of $\theta$ and then the evaluation function $(V)$ can be identified as:

$$
V\left(\theta_{2}, \theta_{4}, T_{e}, W, \theta^{r e f}\right)=\left\|W\left(\theta^{r e f}-\theta\right)\right\|
$$

The evaluation function $(V)$ can be minimized using $\theta_{2}$ and $\theta_{4}$ and expressed as:

$$
\arg V_{\min }\left(\begin{array}{c}
\left.\theta_{2}, \theta_{4}, T_{e}, W, \theta^{r e f}\right) \\
\theta_{2}, \theta_{4}
\end{array}\right.
$$

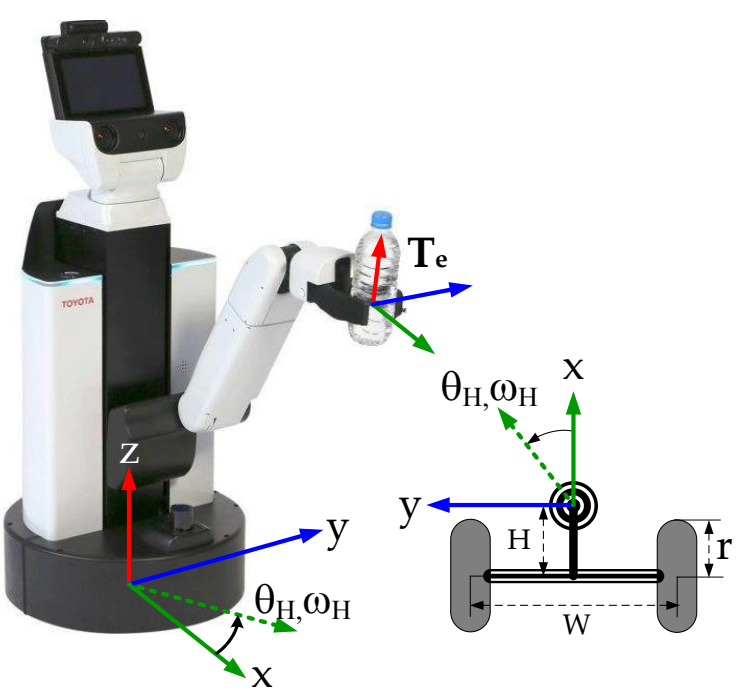

(a)

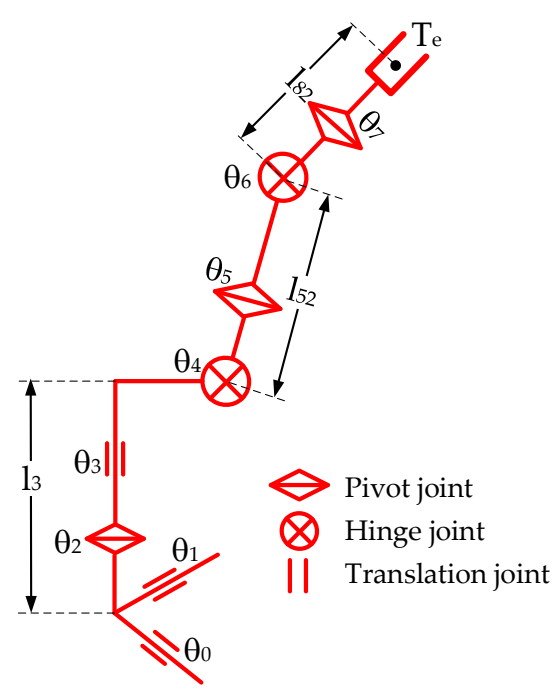

(b)

Figure 3. (a) The dual-wheel caster-drive robot base and (b) the robot join configuration.

\subsection{Coordinate Registration in the HRH System}

This section proposes all coordinate registration for the HRH system. It consists of the robot base frame (robot reference frame) [R], the robot end-effector frame [A], object (held by the robot) frame [T], RGBD camera frame [C], human frame $[\mathrm{H}]$ and handover location frame [L]. All relative frames in the HRH system are drawn in Figure 4. It is to be noted that all the reference frames are in Cartesian coordinates. According to the human frame $[\mathrm{H}]$ can be located by a tracking control based on the head_pose_estimation package [42] as mentioned previously. A human head is identified based on a personalized template by the spherical kernel using a supervised learning algorithm, namely a discriminative random regression forests (DRRF) technique. The human nose tip vector is a crucial aspect that can describe the changes of the head pose neatly. Subsequently, the nose tip frame is appropriately used as the human reference frame [H]. Then, it can be noted that the handover location frame [L] is associated with the human (nose tip) frame [H].

The frame relationship between the object held by the robot and the human hand is the chief requirement implemented to successfully control the robot movements in the HRH tasks. The Transformation matrix has been investigated to identify the posture of the object [T] held by the HSR end-effector [A] relative to the robot reference frame [R]. It can be implied that the robot should manipulate the held object to the receiver naturally at the handover location (incorporating Equation (1)). The Transformation matrix of the handover location frame [L] relative to the robot base frame [R] can be determined based on (1) the 
$[\mathrm{L}]$ referenced by the $[C]$ and (2) the [L] referenced via [R]. Hence, their relationship can be written as Equation (14).

$$
{ }_{T}^{R} T={ }_{A}^{R} T \cdot{ }_{T}^{A} T={ }_{C}^{R} T \cdot{ }_{H}^{C} T \cdot{ }_{L}^{H} T \cdot{ }_{T}^{L} T
$$

where ${ }_{j}^{i} T$ denotes the coordinate transformation from the $j$ frame relative to the $i$ frame. The ${ }_{T}^{R} T$ can be solved using the robot forward kinematics equations. The ${ }_{H}^{C} T$ can be measured by the tracking system. The ${ }_{C}^{R} T$ can be adopted from the robot configuration and ${ }_{L}^{H} T$ can be calculated using Equation (1) conducted by the HHH experiments. Finally, the ${ }_{T}^{L} T$ can be computed using the following equation:

$$
{ }_{T}^{L} T=\left({ }_{L}^{H} T\right)^{-1} \cdot\left({ }_{H}^{C} T\right)^{-1} \cdot\left({ }_{C}^{R} T\right)^{-1} \cdot{ }_{A}^{R} T \cdot{ }_{T}^{A} T
$$

The transformation matrix of the [L] frame relative to the $[R]$ can be expressed by Equation (16).

$$
{ }_{L}^{R} T={ }_{A}^{R} T \cdot{ }_{T}^{A} T \cdot\left({ }_{T}^{L} T\right)^{-1}
$$

The relationship is subsequently implemented on the real-time hybrid PID position and impedance control of the robot compliant motions in HRH.

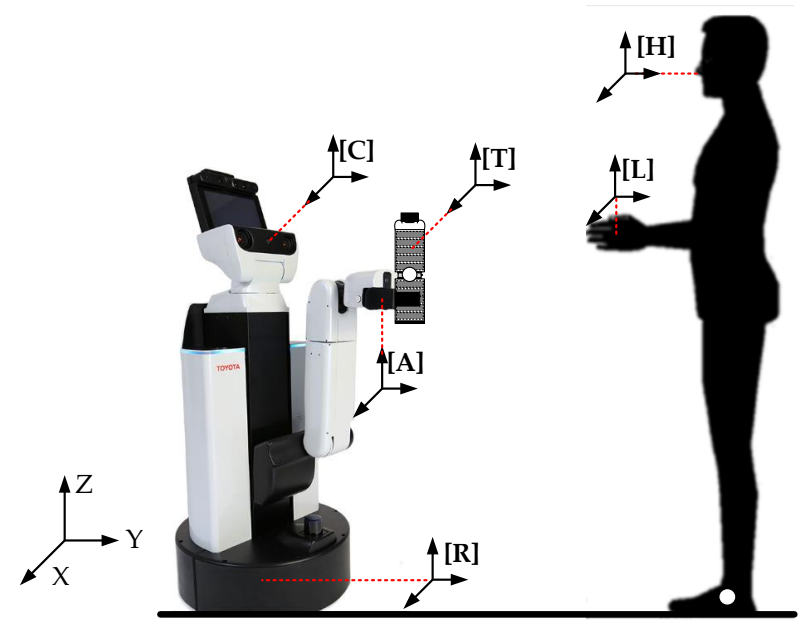

Figure 4. Reference frames of the HRH system.

\subsection{Robotic Hybrid Position/Force Control Strategy for the HRH Tasks}

Robot force control is a fundamental requirement in the achievement of the control of the robot's real-time path in any physical HRI task. It has been developed in the past three decades, using for example force, torque and visual feedback to operate robots to suitably participate in unstructured environments. Raibert and Craig [43] introduced a new method for robot force control based on two control loops, consisting of combined position and force control systems, namely hybrid position/force control. One of the crucial advantages of using the hybrid control is that the position and force information is analysed synchronously and independently. These two outputs are combined in the final stage before being converted to drive the robot actuators using joint torque control [44].

In this research, hybrid PID position/impedance control was implemented. This is due to the fact that PID position control is the most widely used and reliable method for controlling a robot manipulator position. Additionally, a method for the automatic tuning of PID controllers in a closed loop can be estimated using a transfer function model. However, the PID controller may not suitable for force control because the derivative term $\left(K_{d}\right)$ is too sensitive to noise. This could induce a destabilizing impact on the robot force control system [45]. Interestingly, Rahman et al. [46] proposed the mathematical models of the human hand while interacting with a HHH task. The human applied force is formulated by the human arm impedance which is made up of mass, stiffness and damping factor, 
respectively. Consequently, to imitate the real-world human force dynamics, impedance control, which efficiently controls both the motion of the robot and its contact forces, was selected in the robotic force control loop.

Figure 5 illustrates the hybrid PID position/impedance control scheme, which was designed based on the study by Vukobratovic et al. [47]. It is made up of two control loops each of which has an individual information sensor system, i.e., (1) multi-axis force/torque sensor used to detect the force applied to the HSR robot end effector and (2) the rotary encoders used to measure the robot joint positions. $X_{R}$ and $F_{R}$ represent the references of the position and force control schemes, which can be generated using handover experimental results, as explained in Equations (1) and (2). The outcomes from the position $\left(X_{P}\right)$ and force control $\left(X_{F}\right)$ loops are fused together as a set of incremental displacements $\left(X_{u}\right)$, namely, the hybrid position/force control, as exhibited in Equation (17). The parameters $X_{E}$ and $F_{E}$ are the errors of the position in Cartesian coordinate and force control algorithms, respectively. The matrix $S$ represents the compliance selection matrix used to specify the number of DOF in the robotic control system, where $A$ and $B$ represent the robotic position and force controllers, respectively.

$$
X_{u}=X_{P}+X_{F}=A\left[\left(S \times X_{E}\right)\right]+B\left[\left((1-S) \times F_{e}\right)\right]
$$

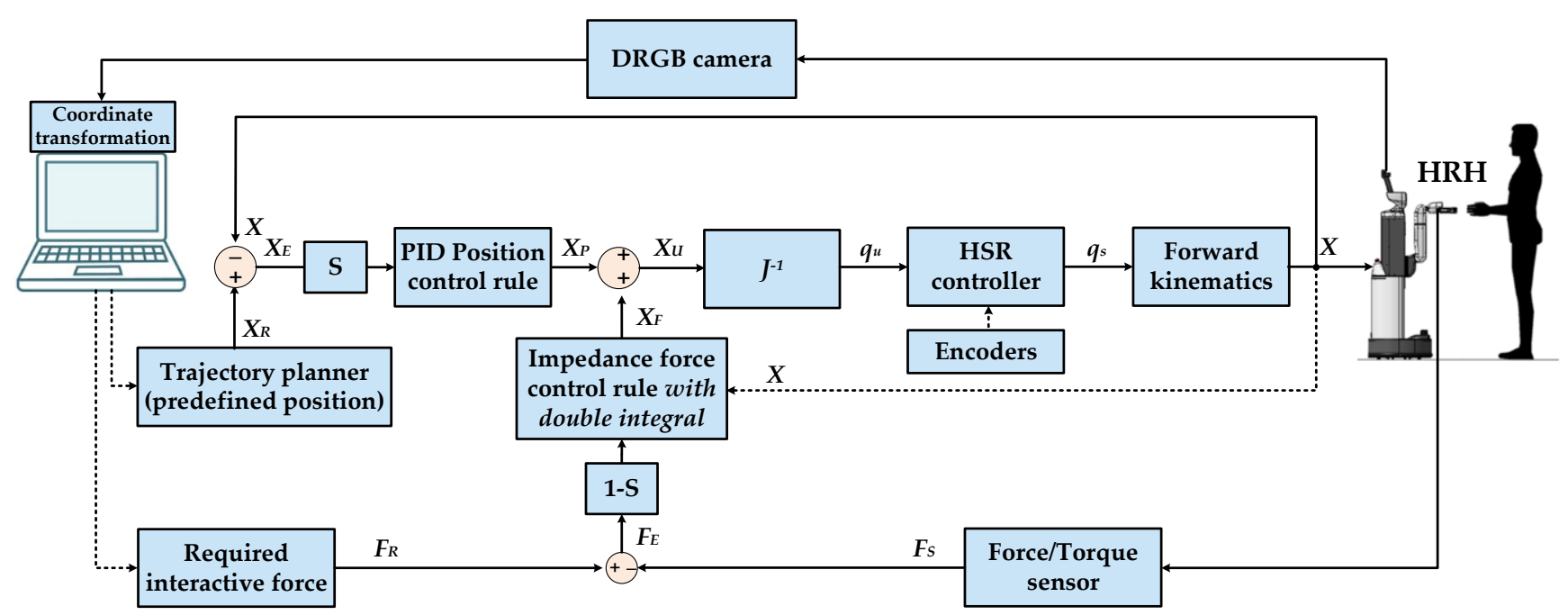

Figure 5. Hybrid PID position/impedance force control designed for the HSR in the HRH tasks.

The positions of the robot in joint space $\left(q_{u}\right)$ can be computed based on an inverse Jacobian matrix as expressed in the following equation.

$$
q_{u}=J(q)^{-1} X_{u}
$$

The position feedback of the robot in Cartesian space $(X)$ is adopted the forward kinematic function based on the position feedback of the robot joint space detected by the encoders $\left(q_{s}\right)$ as formulated in Equation (19).

$$
X=f\left(q_{s}\right)
$$

According to the robot control design, a PID controller was implemented on the robot position control. An incremental discrete-time PID control algorithm with a sampling period $(\tau)$ and the discrete-time interval $k$ was implemented. The robotic position control scheme is written as:

$$
X_{P}(k)=X_{P}(k-1)+(S)\left[k_{p}\left(X_{E}(k)-X_{E}(k-1)\right)+k_{i}\left(X_{E}(k)\right)+k_{d}\left(X_{E}(k)-2 X_{E}(k-1)+X_{E}(k-2)\right)\right]
$$


Additionally, the robot has to interact with a human in $\mathrm{HRH}$, in which the muscles of the human arm can be mechanically represented in a mass-spring and damper system [46]. To facilitate the robotic impedance behaviour, the impedance control approach is therefore executed, which is not only used to track a motion trajectory alone but rather to regulate the mechanical impedance $\left(Z_{m}\right)$ of the robot. The impedance control can be exposed as:

$$
F_{E}=M_{d}\left(\ddot{X}_{F}-\ddot{X}\right)+B_{d}\left(\dot{X}_{F}-\dot{X}\right)+K_{d}\left(X_{F}-X\right)
$$

The real-time trajectory of the robot end-effector can be controlled by acceleration as the following equation; however, the acceleration output incorporates a double integral function before being transmitted to the robot.

$$
\ddot{X}_{F}=\ddot{X}+M_{d}^{-1}\left[F_{E}-B_{d}\left(\dot{X}-\dot{X}_{F}\right)-K_{d}\left(X-X_{F}\right)\right]
$$

where $M_{d}, B_{d}$ and $K_{d}$ represent a designed inertia matrix, damping matrix and stiffness matrix, respectively, which incorporate with $F_{E}$.

\subsection{Dynamic Mechanical Model of the Physical Human-Robot Handover}

This section summarized a mathematical dynamic model of the HRH system. Presumably, the overall interaction can be represented as sub-equivalent-lumped-mass systems as depicted in Figure 6 . The HSR can be modelled by the rigid body model $\left(m_{R}\right)$ associated with effective viscous damping $\left(b_{R}\right)$ to ground and driven by force $F_{R}$ created by the hybrid control algorithm. The robot hand (gripper), which holds the rigid object mass $m_{O}$, has the impedance parameters as $m_{h}, k_{h}$ and $b_{h}$, respectively. The force sensor attached to the hand wrist can be mechanically presented as a spring $\left(k_{s}\right)$ and dashpot $\left(b_{s}\right)$ system. The impedance parameter notation of the human hand comprises the hand mass $\left(m_{H}^{*}\right)$, the viscous damping $\left(b_{H}\right)$ and stiffness $\left(k_{H}\right)$, respectively. In addition, the displacements of the masses $\left(m_{R}, m_{h}\right.$ and $\left.m_{H}\right)$ are, respectively, defined as $x_{R}, x_{h}$ and $x_{H}$, in which it can be assumed as: $m_{H}=m_{O}+m_{H}^{*}$. The state-space equations based modelling representing the overall HRH system can be formulated as:

$$
\begin{aligned}
& \frac{d}{d t}\left[\begin{array}{c}
x_{R} \\
\dot{x}_{R} \\
x_{h} \\
\dot{x}_{h} \\
x_{H} \\
\dot{x}_{H}
\end{array}\right]=\left[\begin{array}{cccccc}
0 & 1 & 0 & 0 & 0 & 0 \\
-\frac{k_{s}}{m_{R}} & -\frac{\left(b_{R}+b_{s}\right)}{m_{R}} & \frac{k_{s}}{m_{R}} & \frac{b_{s}}{m_{R}} & 0 & 0 \\
0 & 0 & 0 & 1 & 0 & 0 \\
\frac{k_{s}}{m_{h}} & \frac{b_{s}}{m_{h}} & -\frac{\left(k_{h}+k_{s}\right)}{m_{h}} & -\frac{\left(b_{h}+b_{s}\right)}{m_{h}} & \frac{k_{h}}{m_{h}} & \frac{b_{h}}{m_{h}} \\
0 & 0 & 0 & 0 & 0 & 1 \\
0 & 0 & \frac{k_{h}}{m_{H}} & \frac{b_{h}}{m_{H}} & -\frac{\left(k_{h}+k_{H}\right)}{m_{H}} & -\frac{\left(b_{h}+b_{H}\right)}{m_{H}}
\end{array}\right]\left[\begin{array}{c}
x_{R} \\
\dot{x}_{R} \\
x_{h} \\
\dot{x}_{h} \\
x_{H} \\
\dot{x}_{H}
\end{array}\right] \\
& +\left[\begin{array}{cccccc}
0 & 0 & 0 & 0 & 0 & 0 \\
\frac{1}{m_{R}} & 0 & 0 & 0 & 0 & 0 \\
0 & 0 & 0 & 0 & 0 & 0 \\
0 & 0 & 0 & 0 & 0 & 0 \\
0 & 0 & 0 & 0 & 0 & 0 \\
0 & 0 & 0 & 0 & 0 & 0
\end{array}\right]\left[\begin{array}{c}
F \\
0 \\
0 \\
0 \\
0 \\
0
\end{array}\right] \text { and } y=\left[\begin{array}{llllll}
1 & 0 & 0 & 0 & 0 & 0 \\
0 & 0 & 1 & 0 & 0 & 0 \\
0 & 0 & 0 & 0 & 1 & 0
\end{array}\right]\left[\begin{array}{c}
x_{R} \\
\dot{x}_{R} \\
x_{h} \\
\dot{x}_{h} \\
x_{H} \\
\dot{x}_{H}
\end{array}\right]+[0] F
\end{aligned}
$$

After taking the Laplace transforms of the state space equations above, the transfer matrices are then given by:

$$
G_{1}(s)=\frac{x_{R}(S)}{F_{R}(S)}=\frac{A}{D}, G_{2}(s)=\frac{x_{h}(S)}{F_{R}(S)}=\frac{B}{D}, G_{3}(s)=\frac{x_{H}(S)}{F_{R}(S)}=\frac{C}{D}
$$

where 

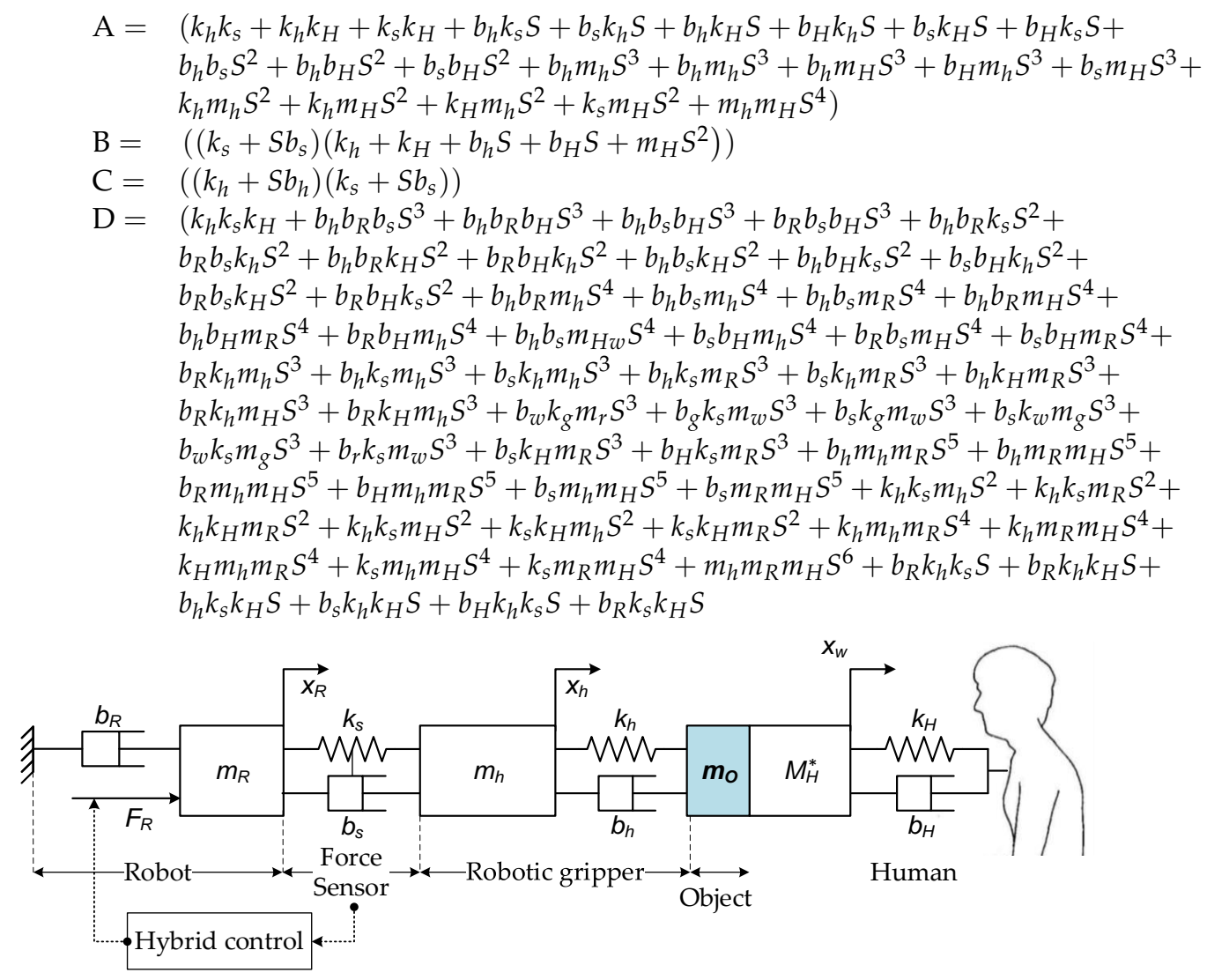

Figure 6. Mechanical schematic of the HRH based on a second-order-lumped-mass model.

\subsection{Safety in $\mathrm{HHH}$}

One of the principal challenges in the development of the HRH task is a safety issue since any failure might become very critical for a human co-worker. Several studies have investigated relative approaches to safety and reliability issues in HRI, i.e., human factors, risk assessment, hazard analysis and technologies for HRI safety, etc. Matthias et al. [48] presented an assessment of the associated risks for possible incidental contact events between a collaborative robot and a human worker based on the guidance published in ISO/TS 15066. The ISO/TS 15066 provides standards of safety and operation for the complex protection schemes required for robots. Many researchers [49-51] proposed conceptual designs to improve safety issues in HRI, such as applying appropriate emergency stops which can be activated as soon as any error occurs, or implementing a validation phase based on control software to facilitate an effective real-time control system etc.

In the robotic system, a safeguarded zone was introduced to conduct a safety strategy that can prevent unauthorised humans from entering the test area. The speed of the HSR movement allowed in the HRI tests was limited and the robot working area was also optimized to minimize the risk of an injury. A timeout, which indicates the period of time allowed for executing a specified task, was assigned for the serial and TCP/IP communication. Safety upper-limit force $\left(f_{\text {lim }}\right)$ was defined. Based on the HHH study [32], the $f_{\text {lim }}$ was recommended to initially set at $10 \mathrm{~N}$ indicating the amount of magnitude of maximum force is taken into account when the handler decides to release the object to be transferred naturally. If the exerted (interactive) force is greater than the limited force, the robot will be immediately terminated. Moreover, the stand-alone emergency stop buttons can be manually activated by a human operator when accidents are detected.

\section{Results and Discussion}

The HHH cooperation has provided a better understanding of what is required in the development of an appropriate set of human-like behaviour for the robot control strategy. 
This can facilitate the dextrous HRH tasks in a safe and effective way. In this research, hybrid PID position and impedance control has been developed and implemented to improve the stability of the robot position and force control. Nevertheless, the key challenge is to suitably design the optimized PID and impedance control algorithms of the HSR, which interacts with the complicated dynamic nature of the human in the object handovers. Subsequently, this section details the evaluation of the performance of the robot control system to facilitate the robot to transfer the object to the human receiver in a safe, timely and natural manner.

\subsection{Optimization of the Robotic PID Position Control}

To successfully adopt an appropriate PID controller, the control gains $\left(k_{p}, k_{i}\right.$ and $k_{d}$ ) can be estimated using the Ziegler-Nichols tuning method incorporated with the robot transfer function of the proposed dynamic model in Section 2.7. However, it is complicated to estimate each model parameter in the dynamic equations and then the MATLAB system identification was consequently applied. One of the effective parametric models identifying system transfer functions which are recommended by Ruslan et al. [52] is the Autoregressive Moving Average eXogenous (ARMAX). The ARMAX is one of the most robust techniques and is normally used to study complex dynamical systems in time-series analysis.

The Z-transform is reasonable for analysis of practical model identification, which presents the relationship between the eigenvalues and the poles of the ARMAX-based estimated model. The model [53] can be formulated as follows:

$$
\begin{aligned}
y(t)+a_{1} y(t-1)+\cdots & +a_{n_{a}} y\left(t-n_{a}\right)=b_{1} u(t-1)+\cdots+b_{n_{b}} u\left(t-n_{b}\right)+e(t) \\
& +c_{1} e(t-1)+\cdots+c_{n_{c}} e\left(t-n_{c}\right)
\end{aligned}
$$

where the observed input and output are represented as $u(k)$ and $y(k)$. The noise signal moderated into the system is $e(k)$.

The Matlab Identification Toolbox was implemented to appropriately estimate the model unknown ARMAX parameters by selecting a set of a polynomial of $n_{a}, n_{b}$ and $n_{c}$ to minimize the forecasting errors and to obtain an effective model. A set of preliminary experiments involving a robot position control system has been conducted. The time-series information required in the system identification is made up of (1) robot position target, namely the ARMAX input and (2) the measured robot position, namely the ARMAX output. Presumably, the range of the robot forward translation movement is approximately 1 meter. Additionally, to present the system-parameter estimation technique under consideration adequately, the model validation finally verifies a proposed identified model. The discretetime ARMAX model was identified and its results are given in Equation (26). The set of minimized polynomial functions is made up of $n_{a}: 2, n_{b}: 2$ and $n_{c}: 2$, respectively.

$$
\begin{aligned}
& A(z)=1+a_{1} z^{-1}+\cdots+a_{n_{a}} z^{-n_{a}}=1-1.993 z^{-1}-0.9934 z^{-2} \\
& B(z)=b_{0}+b_{1} z^{-1}+\cdots+b_{n_{b}} z^{-n_{b}}=0.0001068 z^{-1}-9.555 \mathrm{e}-05 z^{-2} \\
& C(z)=1+c_{1} z^{-1}+\ldots+a_{n_{a}} z^{-n_{c}}=1-0.9241 z^{-1}-0.02802 z^{-2}
\end{aligned}
$$

It is to be noted that the MATLAB discrete-time to continuous-time model conversion was then applied to determine the robotic transfer function into a continuous-time transfer function $\left(H S R_{T F}\right)$ as formulated in Equation (27).

$$
H S R_{T F}=\frac{1.036 \mathrm{e}-05 s^{2}+0.002733 s+0.03113}{s^{2}+1.666 s+0.7003}
$$

Model validation was carried out in the final stage of the model identification process to verify the simulated model. Several model validation techniques were then employed and the results express as follows: (1) percentage of best fit $\left(R^{2}\right)$ was approximately $100 \%$, (2) mean squared error (MSE) was $1.069 \times 10^{-10}$ and finally, (3) final prediction error (FPE) was $1.077 \times 10^{-10}$, respectively. Using the predicted model indicates that the actual and 
estimated data have high similarity in terms of the system responses. Then, it is considered acceptable for the HSR representation model.

After executing Ziegler Nicholas PID tuning in Matlab by providing the numerator and denominator coefficients of the HSR mathematical model in the Laplace domain, it gives gain parameters the critical gain $k_{c}$ and ultimate period $\tau_{c}$. Consequently, it can compute the gain values of $k_{p}, k_{i}$ and $k_{d}$. Nevertheless, the tuning technique still needs an operator's experience for fine-tuning until the system specification is achieved. This offers optimized PID gains as $k_{p}, k_{i}$ and $k_{d}$ of $0.24,0.13$ and 0.02 , respectively. To evaluate the closed-loop stability of the robot system, Figure 7 illustrates the test results and frequency response analysis, whereas the robot moved forwards to the $1 \mathrm{~m}$ target. It can be summarized that a set of the optimized PID gains demonstrates significant satisfaction with the overall control performance in the robotic control system and this is subsequently used in the robot hybrid control approach.

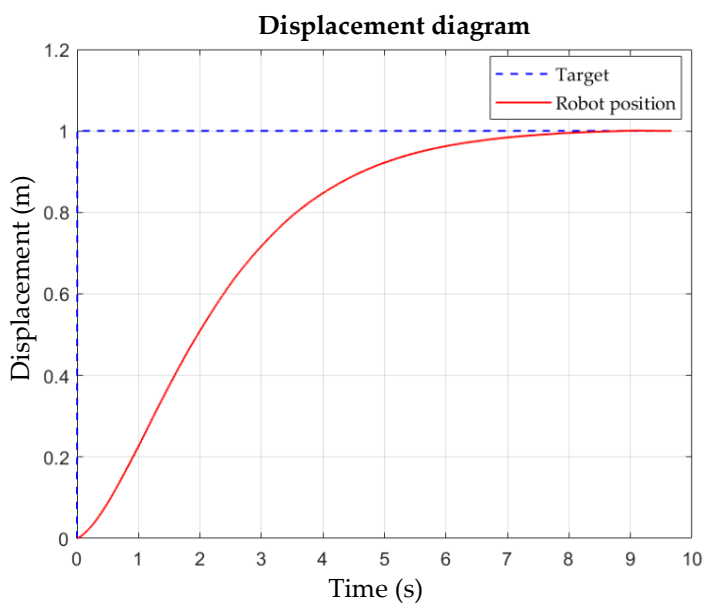

(a)

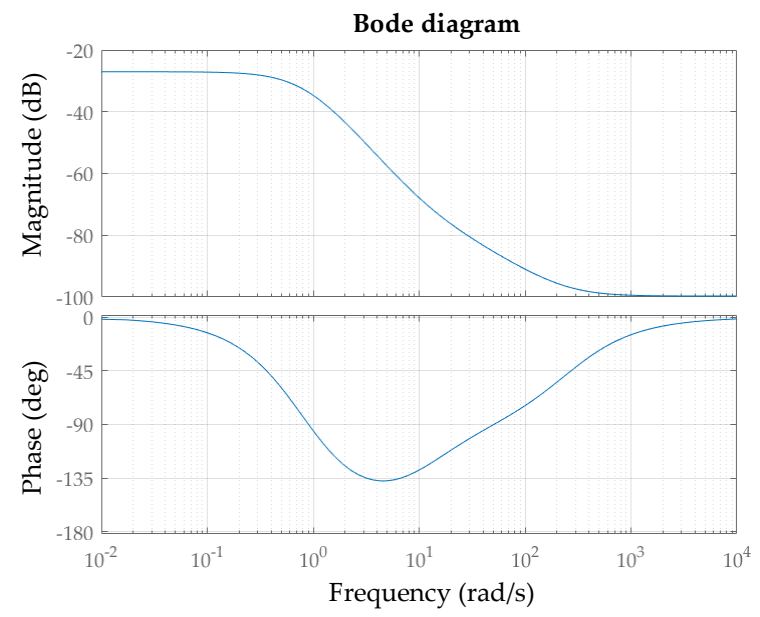

(b)

Figure 7. (a) Robot displacement profile in the HRH test and (b) Bode magnitude and phase plot.

\subsection{Evaluation of the Robotic Impedance Control Based on the HRH}

This section proposes how to achieve the appropriate impedance force control based on the performance evaluations of the robotic dynamic response in the human-to-robot handovers. According to the test procedure, a set of 20 participants has undertaken three repetition sets of each handover test under the required variable conditions. They are defined as a receiver agent and have to grasp the object using one hand and twisting the object is not allowed. The object was fabricated as a bottle-shaped object with $60 \mathrm{~mm}$ diameter, $270 \mathrm{~mm}$ length and a total mass of $1.25 \mathrm{~N}$. The HRH experimental apparatus is shown in Figure 8. The HSR is required to grasp an object and move facing to the object handover location, while the human performs standstill in comfortable positions on opposite sides of the robot. Once the object is manipulated to the transfer location the human is then allowed to grab the object and start naturally pulling the object before taking the responsibility for the object finally. In the meantime, interactive force $\left(F_{\text {int }}\right)$ profiles against time $(t)$ presenting how the robot giver regulates the bilateral force before releasing the object to be transferred to the receiver was measured in real-time.

The circumstances affecting the robot dynamic behaviour compose of spring stiffness $\left(K_{d}\right)$ and damping factor $\left(B_{d}\right)$, whereas $M_{d}$ was strictly specified as a constant parameter of $0.25 \mathrm{Kg}$. Initially, a set of preliminary tests was undertaken to establish the relationship between the interactive force and handover time under various spring stiffness $\left(K_{d}\right)$ and damping factor $\left(B_{d}\right)$ values. The goals of the experiments are to understand how the influential factors affect the interactive forces in the HRH system and to determine the ranges of $B_{d}$ and $K_{d}$ used in the substantive $\mathrm{HRH}$. The test outcomes were demonstrated in Table 1. According to human active comfort, the impedance control system should 
have lower stiffness and damping coefficient values. Conflictingly, in terms of active safety, it should have lower stiffness but a higher damping coefficient. Based on the recommendations of the preliminary study, as the humans generally felt comfortable in the natural physical $\mathrm{HRH}, B_{d}$ should be set as 50 and $75(\mathrm{NmS} / \mathrm{rad})$ and the range of $K_{d}$ should be varied from 0 to $400(\mathrm{Nm} / \mathrm{rad})$, respectively.

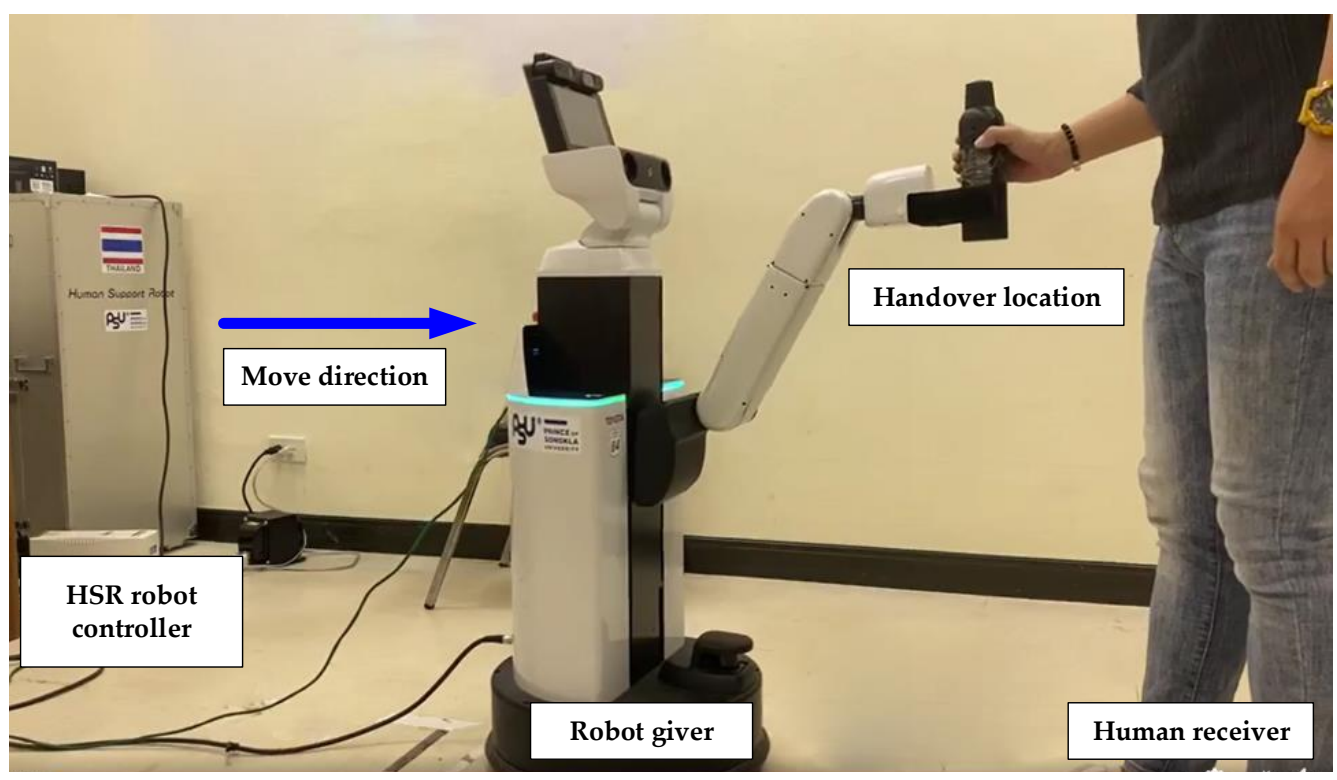

Figure 8. Experimental setup of the robot-to-human object handover system.

Table 1. Comparisons of the average interactive force $\left(F_{i n t}\right)$ and time $(t)$ parameters, while individually varying the damping factor $\left(B_{d}\right)$ and spring stiffness $\left(K_{d}\right)$, respectively.

\begin{tabular}{|c|c|c|c|c|c|c|c|}
\hline \multicolumn{4}{|c|}{ Varied Damping Factor $\left(B_{d}\right)$} & \multicolumn{4}{|c|}{ Varied Spring Stiffness $\left(K_{d}\right)$} \\
\hline$B_{d}(\mathrm{NmS} / \mathrm{rad})$ & $K_{d}(\mathrm{Nm} / \mathrm{rad})$ & $F_{\text {int }}(\mathrm{N})$ & $t(\mathrm{~s})$ & $B_{d}(\mathrm{NmS} / \mathrm{rad})$ & $K_{d}(\mathrm{Nm} / \mathrm{rad})$ & $F_{i n t}(\mathrm{~N})$ & $t(\mathrm{~s})$ \\
\hline 25 & $0(\mathrm{~N} / \mathrm{A})$ & 1.19 & 2.14 & $0(\mathrm{~N} / \mathrm{A})$ & 50 & 0.97 & 3.03 \\
\hline 50 & $0(\mathrm{~N} / \mathrm{A})$ & 1.16 & 2.22 & $0(\mathrm{~N} / \mathrm{A})$ & 100 & 1.00 & 2.99 \\
\hline 75 & $0(\mathrm{~N} / \mathrm{A})$ & 1.12 & 2.32 & $0(\mathrm{~N} / \mathrm{A})$ & 150 & 1.04 & 2.92 \\
\hline 100 & $0(\mathrm{~N} / \mathrm{A})$ & 1.09 & 2.41 & $0(\mathrm{~N} / \mathrm{A})$ & 200 & 1.07 & 2.90 \\
\hline 125 & $0(\mathrm{~N} / \mathrm{A})$ & 1.05 & 2.49 & $0(\mathrm{~N} / \mathrm{A})$ & 300 & 1.16 & 2.84 \\
\hline 150 & $0(\mathrm{~N} / \mathrm{A})$ & 1.00 & 2.61 & $0(\mathrm{~N} / \mathrm{A})$ & 400 & 1.20 & 2.80 \\
\hline
\end{tabular}

The substantive HRH experiments were successfully carried out by the 20 selected subjects. A comparison of the average interactive force and handover time under the several conditions based on the HRH tasks is given in Table 2. After careful observation with regard to the HRH test results, it was complicated to select the set of optimized impedance parameters as its nature necessitates human judgments. After asking the participants, two desirable choices of the impedance parameters were selected based on the recommendations, which consist of Set 1: $B_{d}(50), K_{d}(200)$ and $M_{d}(0.25)$ and Set 2: $B_{d}(75), K_{d}(50)$ and $M_{d}(0.25)$, as highlighted in Table 2. A questionnaire technique was subsequently employed to gather information from the human participants. Rating scale questions were introduced and specified with the five-point rating scale from 1 (very dissatisfied) to 5 (very satisfied). The two questions were used to ask the participants after participating in the $\mathrm{HHH}$ and $\mathrm{HRH}$ based on both impedance parameter sets as follows:

(1) How do you compare the qualitative performance of the $\mathrm{HHH}$ to that of the $\mathrm{HRH}$ using the parameter Set 1 ? 
(2) How do you compare the qualitative performance of the $\mathrm{HHH}$ to that of the $\mathrm{HRH}$ using the parameter Set 2?

Table 2. Comparisons of the average interactive force $\left(F_{\text {int }}\right)$ and time $(t)$ parameters, while varying both damping factor $\left(B_{d}\right)$ and spring stiffness $\left(K_{d}\right)$.

\begin{tabular}{|c|c|c|c|c|c|c|c|}
\hline \multicolumn{4}{|c|}{ Varied Damping Factor $\left(B_{d}\right)$} & \multicolumn{4}{|c|}{ Varied Spring Stiffness $\left(K_{d}\right)$} \\
\hline$B_{d}(\mathrm{NmS} / \mathrm{rad})$ & $K_{d}(\mathrm{Nm} / \mathrm{rad})$ & $F_{\text {int }}(\mathrm{N})$ & $t(\mathrm{~s})$ & $B_{d}(\mathrm{NmS} / \mathrm{rad})$ & $K_{d}(\mathrm{Nm} / \mathrm{rad})$ & $F_{\text {int }}(\mathrm{N})$ & $t(\mathrm{~s})$ \\
\hline 50 & 0 & 0.96 & 1.85 & 75 & 0 & 1.21 & 1.62 \\
\hline 50 & 50 & 1.24 & 2.02 & 75 & 50 & 1.31 & 1.86 \\
\hline 50 & 100 & 1.19 & 1.73 & 75 & 100 & 1.11 & 2.06 \\
\hline 50 & 200 & 1.31 & 1.36 & 75 & 200 & 1.32 & 2.14 \\
\hline 50 & 300 & 1.25 & 1.51 & 75 & 300 & 1.41 & 1.93 \\
\hline 50 & 400 & 1.11 & 1.42 & 75 & 400 & 1.30 & 1.37 \\
\hline
\end{tabular}

Figure 9 depicts the responses of the participants comparing the overall stability of the robot-human handovers using the different sets of the impedance parameters. It can be observed that 11 participants (55\%) and three participants (15\%), respectively, were significantly satisfied and very satisfied with the overall stability of the robot handover system using the first parameter set. The second set shows $12(60 \%)$ and $6(30 \%)$ subjects were satisfied and very satisfied. There is only one person $(5 \%)$ very dissatisfied with the parameter set 1 , while another leads participants to experience the robot handover without any dissatisfaction. To compare the mean measurements of the two set undertaken from the same sample group, the paired samples t-test was statistically utilized. The following hypotheses $H_{0}$ and $H_{1}$ were tested as:

$H_{0}: \mu_{\text {Set } 2}=\mu_{\text {Set } 1}$, which means the median difference between pairs of observations is zero so that there is no significant difference in the population distributions.

$H_{0}: \mu_{\text {Set } 2}>\mu_{\text {Set } 1}$, which means the median difference between pairs of observations is not equal to zero and the population rating from set 2 is greater than that of set 1 .

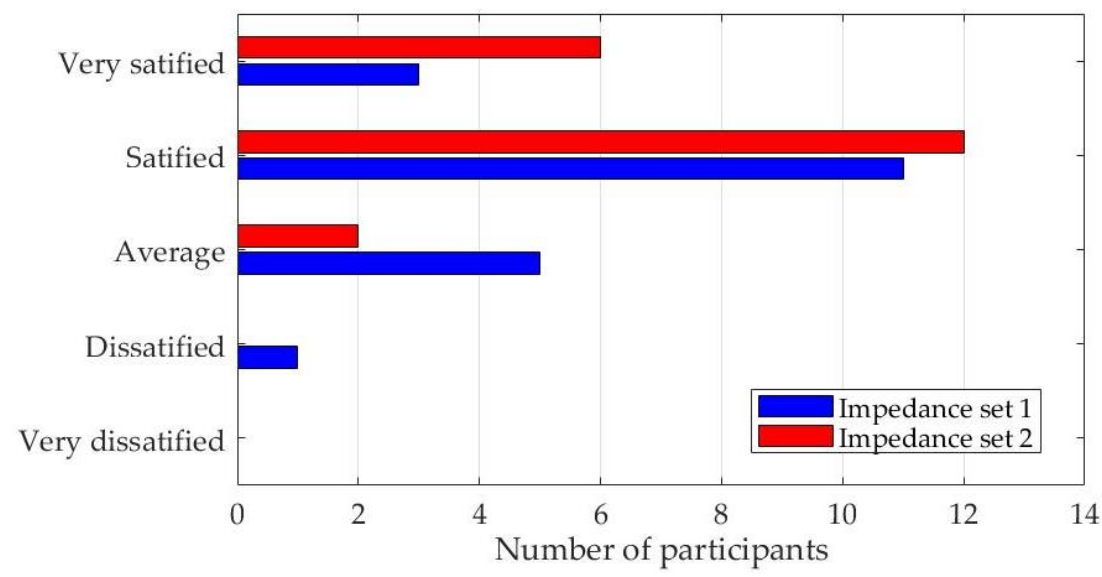

Figure 9. Survey responses for the human-robot handover tasks.

The results of the statistical analysis based on the paired $t$-test present that ratings of the robot performance based on the parameter set 1 and 2 were 3.80 and 4.20, respectively, with corresponding standard deviations of 0.768 and 0.616 , as summarized in Table 3. This represents the test subjects were more appreciative of the performance of the robot behavioural control using the second parameter set rather than the first set. The value of the paired-sample test was -3.559 (2-tails significance $=0.002)$. It can be described that the hypothesis $H_{0}$ has to be statistically rejected and the alternative hypothesis $H_{1}$ accepted. This means that there is a significant difference in the population distributions from the participants. Additionally, the population rating from set 2 is greater than that of set 1 , in which the participants were more comfortable with the robot's stability using the 
impedance parameters made up of $K_{d}(50), B_{d}(75)$ and $M_{d}(0.25)$, more than those of the first set.

Table 3. T-test results of the comparison between the parameter set 1 and 2 .

\begin{tabular}{|c|c|c|c|c|c|c|c|c|c|}
\hline \multicolumn{10}{|c|}{ Paired Samples Statistics } \\
\hline & & \multicolumn{2}{|c|}{ Mean } & $\mathrm{N}$ & \multicolumn{2}{|r|}{ SD } & \multicolumn{3}{|c|}{ SD Error Mean } \\
\hline \multirow{2}{*}{ Pair 1} & Impedence1 & \multicolumn{2}{|c|}{3.80} & 20 & \multicolumn{2}{|r|}{0.768} & \multicolumn{3}{|c|}{0.172} \\
\hline & Impedence2 & \multicolumn{2}{|c|}{4.20} & 20 & \multicolumn{2}{|r|}{0.616} & \multicolumn{3}{|c|}{0.138} \\
\hline \multicolumn{10}{|c|}{ Paired Samples Correlations } \\
\hline & & & & $\mathrm{N}$ & \multicolumn{2}{|c|}{ Correlation } & \multicolumn{3}{|c|}{ Sig. } \\
\hline Pair 1 & \multicolumn{3}{|c|}{ Impedence1 \& Impedence2 } & 20 & \multicolumn{2}{|r|}{0.757} & \multicolumn{3}{|c|}{0.000} \\
\hline \multicolumn{10}{|c|}{ Paired Samples Test } \\
\hline & & \multicolumn{5}{|c|}{ Paired Differences } & \multirow{3}{*}{$\mathrm{t}$} & \multirow{3}{*}{$\mathrm{df}$} & \multirow{3}{*}{$\begin{array}{c}\text { Sig. } \\
\text { (2-tailed) }\end{array}$} \\
\hline & & \multirow{2}{*}{ Mean } & \multirow{2}{*}{ SD } & \multirow{2}{*}{$\begin{array}{l}\text { SD Error } \\
\text { Mean }\end{array}$} & \multicolumn{2}{|c|}{ 95\% Confidence of the Difference } & & & \\
\hline & & & & & Lower & Upper & & & \\
\hline Pair 1 & $\begin{array}{l}\text { Impedence1 \& } \\
\text { Impedence2 }\end{array}$ & -0.400 & 0.503 & 0.112 & -0.635 & -0.165 & -3.559 & 19 & 0.002 \\
\hline
\end{tabular}

Finally, the results of the robotic dynamic response based on the hybrid PID position and impedance control in the robot-to-human handovers were carried out and then compared with the outcomes of the HHH. Figure 10 shows an example of a comparison between the interactive force profiles against time from the $\mathrm{HHH}$ and HRH tests. It can be concluded that the quantitative measurement of the performance of robot behavioural control can be considered acceptable for object handover interaction with a human receiver. It provided effective performance during the natural cooperative tasks, where the robot was able to successfully pass the object to the human in a safe, reliable and timely manner. However, after careful analysis with regard to the HRH results, the robot has to perform a slightly longer handover period than that of the HHI, even though the maximum forces from both cases are similar. This is because the robot control algorithm was initially designed to ensure its safe and natural operational characteristics specifically while physically releasing the object to the receiver. Then, this might result longer object handover process.

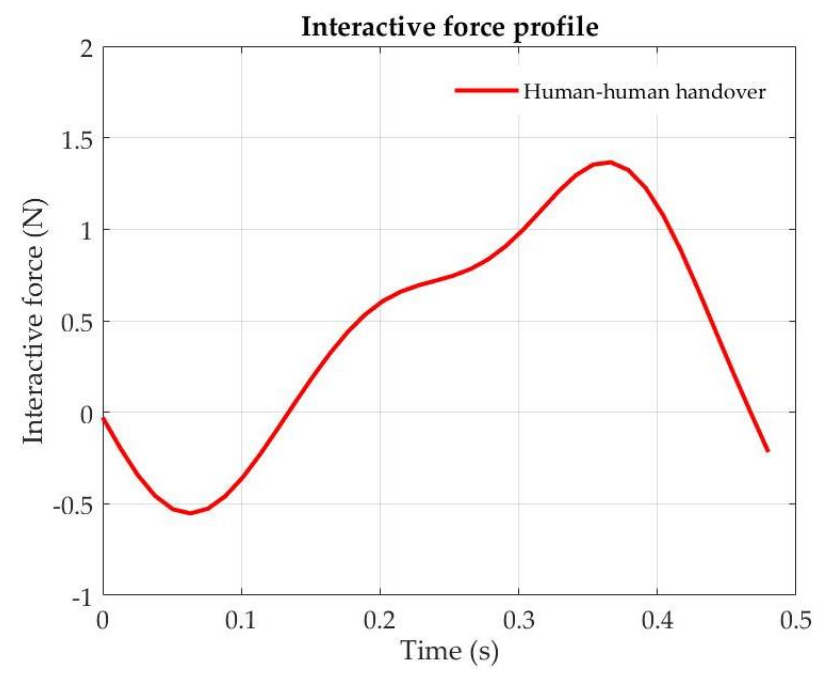

(a)

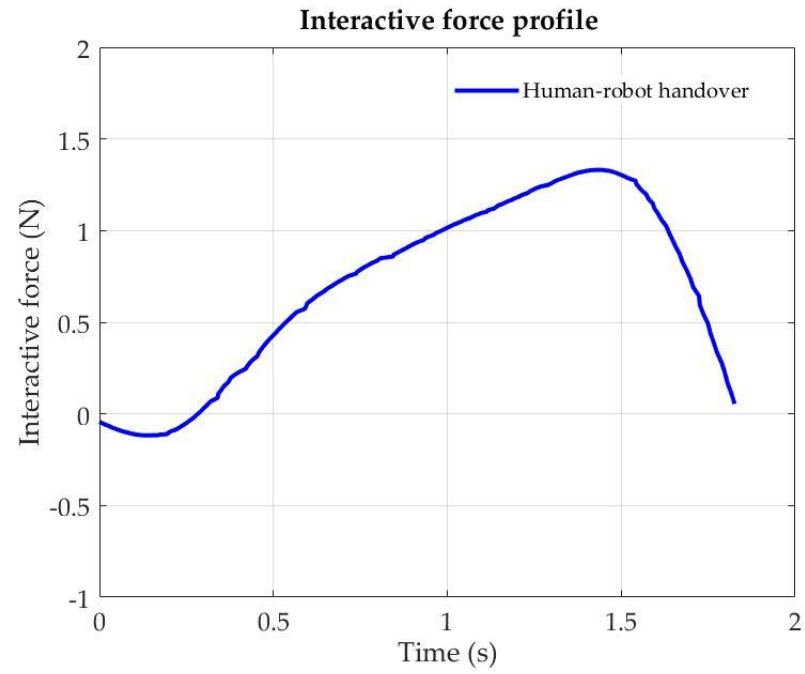

(b)

Figure 10. Interactive force profiles against the time of (a) the HHH test and (b) the HRH test. 
Consequently, it can be concluded that understanding kinematically and dynamically how two humans physically collaborate while naturally performing object handover tasks offers the effective performance and reliability of the robot-to-human handover procedure and acceptable HRI implementation. This was in agreement with the parallel test outcomes, demonstrating significant satisfaction with the overall performance of the HRH system, as measured by an average rating of 4.20 on a five-point scale.

\section{Conclusions}

This paper contributes to the implementation of a human-like behavioural control strategy for seamless HRH. The robotic conceptual framework was established by understanding the principle of human haptic interaction when two humans work together in a joint effort to complete an object handover task. The performance of the robot-human handover system has been evaluated based on the interactive fore profiles, handover time and how comfortable the participants felt in participating in the HRH tasks. The results provide effective performance during the HRH, where the HSR robot was able to successfully pass the object to the human effectively. The safety systems proposed are working successfully and thus avoiding the likelihood of unsafe HRH actions being taken. Moreover, the feedback responses from the human subjects participating in the HRI also agreed with the parallel test results that gave more satisfaction with a higher average rating scale.

Therefore, it can be concluded that the robot based on the developed behavioural control algorithm is capable of tracking the object transfer location, manipulating the object and handling it to the receiver in a safe, reliable and dexterous manner. This developed robotic system can enhance the accuracy of positioning and force regulating abilities in the HRH system and also increase the smoothness of physical HRI. The outcomes of this research can successfully play a beneficial role in the daily lives of people in future.

Author Contributions: Conceptualization, P.N.; methodology, P.N. and T.S.; software, T.S.; validation, P.N. and T.S.; formal analysis, P.N. and T.S.; investigation, P.N. and T.S.; resources, P.N.; data curation, P.N. and T.S.; writing-original draft preparation, P.N.; writing-review and editing, P.N.; visualization, P.N.; supervision, P.N.; project administration, P.N.; funding acquisition, P.N. All authors have read and agreed to the published version of the manuscript.

Funding: This research received no external funding.

Institutional Review Board Statement: The study was conducted according to the guidelines of the Declaration of Helsinki.

Informed Consent Statement: Informed consent was obtained from all subjects involved in the study.

Data Availability Statement: Not applicable.

Conflicts of Interest: The authors declare no conflict of interest.

\section{References}

1. Goodrich, M.; Schultz, A. Human-Robot Interaction: A Survey. Found. Trends Hum. Comput. Interact. 2007, 1, 203-275. [CrossRef]

2. Shafkat Tanjim, M.S.; Rafi, S.A.; Oishi, A.N.; Barua, S.; Dey, H.C.; Babu, M.R. Image Processing Intelligence Analysis for Robo-Res 1.0: A Part of Humanoid Rescue-Robot. In Proceedings of the 2020 IEEE Region 10 Symposium (TENSYMP), Dhaka, Bangladesh, 5-7 June 2020; pp. 1229-1232.

3. Zhao, J.; Gao, J.; Zhao, F.; Liu, Y. A Search-and-Rescue Robot System for Remotely Sensing the Underground Coal Mine Environment. Sensors 2017, 17, 2426. [CrossRef] [PubMed]

4. Cardona, G.A.; Calderon, J.M. Robot Swarm Navigation and Victim Detection Using Rendezvous Consensus in Search and Rescue Operations. Appl. Sci. 2019, 9, 1702. [CrossRef]

5. e Silva, L.C.; Daher, S.F.D.; Santiago, K.T.M.; Costa, A.P.C.S. Selection of an Integrated Security Area for locating a State Military Police Station based on MCDM/A method. In Proceedings of the 2019 IEEE International Conference on Systems, Man and Cybernetics (SMC), Bari, Italy, 6-9 October 2019; pp. 1530-1534.

6. Boisboissel, G. Is it sensible to grant autonomous decision-making to military robots of the future? In Proceedings of the 2017 International Conference on Military Technologies (ICMT), Brno, Czech Republic, 31 May-2 June 2017; pp. 738-742. 
7. Rosli, R.; Abdullah, M.; Siregar, N.C.; Hamid, N.S.A.; Abdullah, S.; Beng, G.K.; Halim, L.; Daud, N.M.; Bahari, S.A.; Majid, R.A.; et al. Exploring Space Science through the UKM-SIDn Outreach Program. In Proceedings of the 2019 International Conference on Space Science and Communication (IconSpace), Johor Bahru, Malaysia, 28-30 July 2019; pp. 253-256.

8. Xing, Z.; Zhao, Y.; Zhu, S. Path Planning Method Design and Dynamic Model Simplification of Free-Flying Space Robot. In Proceedings of the 2020 15th IEEE Conference on Industrial Electronics and Applications (ICIEA), Kristiansand, Norway, 9-13 November 2020; pp. 1501-1505.

9. Islam, M.R.; Chowdhury, F.H.; Rezwan, S.; Ishaque, M.J.; Akanda, J.U.; Tuhel, A.S.; Riddhe, B.B. Novel design and performance analysis of a Mars exploration robot: Mars rover mongol pothik. In Proceedings of the 2017 Third International Conference on Research in Computational Intelligence and Communication Networks (ICRCICN), Kolkata, India, 3-5 November 2017; pp. 132-136.

10. Javaid, M.; Estivill-Castro, V. Explanations from a Robotic Partner Build Trust on the Robot's Decisions for Collaborative Human-Humanoid Interaction. Robotics 2021, 10, 51. [CrossRef]

11. Katsanis, I.A.; Moulianitis, V.C. An Architecture for Safe Child-Robot Interactions in Autism Interventions. Robotics 2021, 10, 20. [CrossRef]

12. Mi, J.; Takahashi, Y. Whole-Body Joint Angle Estimation for Real-Time Humanoid Robot Imitation Based on Gaussian Process Dynamical Model and Particle Filter. Appl. Sci. 2020, 10, 5. [CrossRef]

13. Jung, S.E.; Won, E.-S. Systematic Review of Research Trends in Robotics Education for Young Children. Sustainability 2018, 10, 905. [CrossRef]

14. Aa Diyas, Y.; Brakk, D.; Aimambetov, Y.; Sandygulova, A. Evaluating peer versus teacher robot within educational scenario of programming learning. In Proceedings of the 2016 11th ACM/IEEE International Conference on Human-Robot Interaction (HRI), Christchurch, New Zealand, 7-10 March 2016; pp. 425-426.

15. Stuhlenmiller, F.; Weyand, S.; Jungblut, J.; Schebek, L.; Clever, D.; Rinderknecht, S. Impact of Cycle Time and Payload of an Industrial Robot on Resource Efficiency. Robotics 2021, 10, 33. [CrossRef]

16. Tao, Y.; Chen, C.; Wang, T.; Chen, Y.; Xiong, H.; Ren, F.; Zou, Y. A Re-Entry Path Planning Method for Service Robots Based on Dynamic Inver-Over Evolutionary Algorithm. Appl. Sci. 2020, 10, 305. [CrossRef]

17. Kupcsik, A.; Hsu, D.; Lee, W.S. Learning Dynamic Robot-to-Human Object Handover from Human Feedback. In Robotics Research; Bicchi, A., Burgard, W., Eds.; Springer Proceedings in Advanced Robotics; Springer: Berlin/Heidelberg, Germany, 2018; Volume 2, pp. 161-176.

18. Neranon, P. Implicit force control approach for Safe physical Robot-to-Human object handover. Indones. J. Electr. Eng. Comput. Sci. 2020, 17, 615-628. [CrossRef]

19. He, W.; Sidobre, D. Improving human-robot object exchange by online force classification. J. Hum. Robot Interact. 2015, 4, 75-94. [CrossRef]

20. Strabala, K.; Lee, M.K.; Dragan, A.; Forlizzi, J.; Srinivasa, S.S.; Cakmak, M.; Micelli, V. Toward Seamless Human-Robot Handovers. J. Hum. Robot Interact. 2013, 2, 112-132. [CrossRef]

21. Emmelmann, M. Influence of velocity on the handover delay associated with a radio-signal-measurement-based handover decision. In Proceedings of the VTC-2005-Fall. 2005 IEEE 62nd Vehicular Technology Conference, Dallas, TX, USA, 28-30 September 2005; pp. 2282-2286.

22. Neranon, P. Human-to-Robot Object Handover using a Behavioural Position-based Force Control Approach. In Proceedings of the First International Symposium on Instrumentation, Control, Artificial Intelligence, and Robotics (ICA-SYMP), Bangkok, Thailand, 16-18 January 2019; pp. 5-8. [CrossRef]

23. Huber, M.; Radrich, H.; Wendt, C.; Rickert, M.; Knoll, A.; Brandt, T. Evaluation of a novel biologically inspired trajectory generator in human-robot interaction. In Proceedings of the RO-MAN 2009-The 18th IEEE International Symposium on Robot and Human Interactive Communication, Toyama, Japan, 27 September-2 October 2009; pp. 639-644.

24. Kajikawa, S.; Okino, T.; Ohba, K.; Inooka, H. Motion planning for hand-over between human and robot. In Proceedings of the 1995 IEEE/RSJ International Conference on Intelligent Robots and Systems, Pittsburgh, PA, USA, 5-9 August 1995; pp. 193-199.

25. Chan, W.P.; Parker, C.A.; Van der Loos, H.F.; Croft, E.A. Grip forces and load forces in handovers: Implications for designing human-robot handover controllers. In Proceedings of the 7th Annual ACM/IEEE International Conference on Human-Robot Interaction, Boston, MA, USA, 2-5 March 2012; pp. 9-16.

26. Baier, T.; Zhang, J. Reusability-based semantics for grasp evaluation in context of service robotics. Robotics and Biomimetics. In Proceedings of the 2006 IEEE International Conference on Robotics and Biomimetics (ROBIO'06), Kunming, China, 17-20 December 2006; pp. 703-708.

27. Nemlekar, H.; Dutia, D.; Li, Z. Object Transfer Point Estimation for Fluent Human-Robot Handovers. In Proceedings of the 2019 International Conference on Robotics and Automation (ICRA), Montreal, QC, Canada, 20-24 May 2019; pp. 2627-2633. [CrossRef]

28. Lemic, F.; Behboodi, A.; Famaey, J.; Mathar, R. Location-Based Discovery and Vertical Handover in Heterogeneous Low-Power Wide-Area Networks. IEEE Internet Things J. 2019, 6, 10150-10165. [CrossRef]

29. Cakmak, M.; Srinivasa, S.; Lee, M.K.; Forlizzi, J.; Kiesler, S. Human preferences for robot-human hand-over configulations. In Proceedings of the 2011 IEEE/RSJ International Conference on Intelligent Robots and Systems (IROS), San Francisco, CA, USA, 25-30 September 2011; pp. 1986-1993. 
30. Chan, W.P.; Pan, M.K.; Croft, E.A.; Inaba, M. Characterization of handover orientations used by humans for efficient robot to human handovers. In Proceedings of the 2015 IEEE/RSJ International Conference on Intelligent Robots and Systems (IROS), Hamburg, Germany, 28 September-2 October 2015; pp. 1-6.

31. Aleotti, J.; Micelli, V.; Caselli, S. An affordance sensitive system for robot to human object handover. Int. J. Soc. Robot. 2014, 6, 653-666. [CrossRef]

32. Sutiphotinun, T.; Neranon, P.; Vessakosol, P.; Romyen, A.; Hiransoog, C.; Sookgaew, J. A Human-Inspired Control Strategy: A Framework for Seamless Human-Robot Handovers. J. Mech. Eng. Res. Dev. 2020, 43, 235-245.

33. Kato, S.; Yamanobe, N.; Venture, G.; Yoshida, E.; Ganesh, G. The where of handovers by humans: Effect of partner characteristics, distance and visual feedback. PLoS ONE 2019, 14, e0217129. [CrossRef]

34. Chien, M.H.; Maya, C.; Bilge, M. Adaptive Coordination Strategies for Human-Robot Handovers. In Proceedings of the Robotics: Science and Systems, Rome, Italy, 13-17 July 2015; pp. 1-10.

35. Sisbot, E.A.; Alami, R. A human-aware manipulation planner. IEEE Trans. Robot. 2012, 28, 1045-1057. [CrossRef]

36. Glasauer, S.; Huber, M.; Basili, P.; Knoll, A.; Brandt, T. Interacting in time and space: Investigating human-human and humanrobot joint action. In Proceedings of the 19th International Symposium in Robot and Human Interactive Communication, Viareggio, Italy, 13-15 September 2010; pp. 252-257. [CrossRef]

37. Kim, J.; Park, J.; Hwang, Y.K.; Lee, M. Advanced Grasp Planning for Handover Operation Between Human and Robot: Three Handover Methods in Esteem Etiquettes Using Dual Arms and Hands of Home-Service Robot. In Proceedings of the 2nd International Conference on Autonomous Robots and Agents, Palmerston North, New Zealand, 12-14 December 2004; pp. 34-39.

38. Huber, M.; Rickert, M.; Knoll, A.; Brandt, T.; Glasauer, S. Human-robot interaction in handing-over tasks. Robot and Human Interactive Communication, 2008. RO-MAN 2008. In Proceedings of the 17th IEEE International Symposium, Munich, Germany, 1-3 August 2008; pp. 107-112.

39. Jae-Bong, Y.; Taewoong, K.; Dongwoon, S.; Seung-Joon, Y. Unified Software Platform for Intelligent Home Service Robots. Appl. Sci. 2020, 10, 5874. [CrossRef]

40. Denavit, J.; Hartenberg, R.S. A kinematic notation for lower-pair mechanisms based on matrices. J. Appl. Mech. 1955, 22, 215-221.

41. Yamamoto, T.; Terada, K.; Ochiai, A. Development of Human Support Robot as the research platform of a domestic mobile manipulator. Robomech. J. 2019, 6. [CrossRef]

42. Jaroonsorn, P.; Neranon, P.; Smithmaitrie, P.; Dechwayukul, C. Robot-assisted transcranial magnetic stimulation using hybrid position/force control. Adv. Robot. 2020, 34, 1559-1570. [CrossRef]

43. Raibert, M.H.; Craig, J.J. Hybrid Position/Force Control of Manipulators. J. Dyn. Syst. Meas. Control 1981, 103, 126-133. [CrossRef]

44. Volpe, R.; Khosla, P. A theoretical and experimental investigation of explicit force control strategies for manipulators. IEEE Trans. Autom. Control 1993, 38, 1634-1650. [CrossRef]

45. Neranon, P.; Bicker, R. Force/position control of a robot manipulator for human-robot interaction. Therm. Sci. Int. Sci. J. 2016, 20, 537-548. [CrossRef]

46. Rahman, M.M.; Ikeura, R.; Mizutani, K. Investigation of the impedance characteristic of human arm for development of robots to cooperate with humans'. JSME Int. J. Ser. C 2002, 45, 510-518. [CrossRef]

47. Vukobratovic, M.; Surdilovic, D.; Ekalo, Y. Dynamics and Robust Control of Robot-environment Interaction. In New Frontiers in Robotics; World Scientific Publishing Co. Pte. Ltd.: Singapore, 2008; Volume 2.

48. Matthias, B.; Reisinger, T. Example Application of ISO/TS 15066 to a Collaborative Assembly Scenario. In Proceedings of the 47th International Symposium on Robotics: ISR 2016, Munich, Germany, 21-22 June 2016; pp. 1-5.

49. Vasic, M.; Billard, A. Safety Issues in Human-Robot Interactions. In Proceedings of the 2013 IEEE International Conference on Robotics and Automation, Karlsruhe, Germany, 6-10 May 2013; pp. 197-204. [CrossRef]

50. Duchemin, G.; Poignet, P.; Dombre, E.; Peirrot, F. Medically safe and sound [human-friendly robot dependability]. IEEE Robot. Autom. Mag. 2004, 11, 46-55. [CrossRef]

51. Kulic, D.; Croft, E.A. Real-time safety for human-robot interaction. Robot. Auton. Syst. 2006, 54, 1-12. [CrossRef]

52. Ruslan, F.A.; Haron, K.A.; Samad, M.; Adnan, R. Multiple Input Single Output (MISO) ARX and ARMAX model of flood prediction system: Case study Pahang. In Proceedings of the 2017 IEEE 13th International Colloquium on Signal Processing \& Its Applications: CSPA, Penang, Malaysia, 10-12 March 2017; pp. 179-184. [CrossRef]

53. Rabbani, F. Model Identification and Validation for a Heating System using MATLAB System Identification Toolbox. In Proceedings of the 1st International Conference on Sensing for Industry, Control, Communications, \& Security Technologies (ICSICCST 2013), Karachi, Pakistan, 24-26 June 2013. 